Research Article

\title{
Average Sample Number Function for Pareto Heavy Tailed Distributions
}

\author{
Boukhalfa El-Hafsi \\ Department of Mathematics and Computer Science, University of Tebessa, Algeria \\ Correspondence should be addressed to Boukhalfa El-Hafsi; boukhalfa12@yahoo.fr
}

Received 21 April 2013; Accepted 9 May 2013

Academic Editors: S.-W. Chyuan and Q. Song

Copyright ( 2013 Boukhalfa El-Hafsi. This is an open access article distributed under the Creative Commons Attribution License, which permits unrestricted use, distribution, and reproduction in any medium, provided the original work is properly cited.

\begin{abstract}
The main purpose of this work is shortly to give the average sample number function after a sequential probability ratio test on the index parameter alpha of stable densities, which we give a mean of the number of data required to take decision in the case $1<\alpha<2$, we use the fact that the tails of Levy-stable distributions are asymptotically equivalent to a Pareto law for large data. Stable distributions are a rich class of probability distributions that allow skewness and heavy tails and have many intriguing mathematical properties. The lack of closed formulas for densities and distribution functions for all has been a major drawback to the use of stable distributions by practitioners, but few stable distributions have the analytical formula of their densities functions which are Gauss, Levy, and Cauchy.
\end{abstract}

\section{Introduction}

Because it has a large application in many fields, stable distributions have been proposed as a model for many types of physical and economic systems [1-3]. There are several reasons for using a stable distribution to describe a system. The first is where there are solid theoretical reasons for expecting a non-Gausian stable model. The second reason is the Generlized Central Limit Theorem which states that the only possible non-trivial limit of normalized sums of independent identically distributed terms is stable. The third argument for modeling with stable distributions is empirical: many large data sets exhibit heavy tails and skewness. The strong empirical evidence for these features combined with Generalized Central Limit Theorem is used by many to justify the use of stable models. There are now reliable computer programs to compute stable densities, distribution functions and quantiles. With these programs, it is possible to use stable models in variety of practical problems $[1,2]$.

In Section 1, we give some definitions, arithmetic properties of stable laws, and the numerical calculation of stable densities, distribution functions Mittnik et al. [4] and
Nolan [1, 2], and simulation algorithm [1,5]. In Section 2, we propose a method to calculate the likelihood ratio appliying the pareto limit of stable densities for large data, with discuss, Finally we interests of the number of observations needed to take decision about the best value of index parameter by its averange sample number function in the case $1<\alpha<2$, when moment of order one exists.

There are now reliable computer programs to compute stable densities, distribution functions and quantiles. Nolan [2] creates algorithms for generate general stable densities based on integral representations of the densities which were derived by Zolotarev [6], then Nolan [2,3] provides a useful program named "STABLE". With these program, it is possible to use stable models in variety of practical problems. For Wald's SPRT [7] approximatios, Raghavachari [8] gave exact formulas in the case of an exponential density $\theta^{-1} \exp (-x / \theta)$ for testing $\theta_{0}$ versus $\theta_{1}$, provided $\theta_{0} / \theta_{1}>\max (B, B / A)$. For a sequential probability ratio test, Under other distributions $A$. Wald's approximations [7] are often used, but how about stable distributions? there are not closed formula for the densities to calculate the likelihood ratio, we compare the fit of two pareto stable models using sequential probability ratio test. 


\section{Stable Distributions}

Definition 1. A random variable $X$ has a distribution infinitely divisible if and only if for all $n, \exists X_{1}, \ldots, X_{n}$ independent with the same even law such as

$$
X \stackrel{d}{=} X_{1}+\cdots+X_{n}
$$

where $\stackrel{d}{=}$ means equality in distribution.

Remark 2. The random variables $X_{i}$ did not even law as $X$. However, as we see in the following examples, they belong to the same family of distributions.

Example 3. The characteristic function of normal distribution $\mathcal{N}\left(\mu, \sigma^{2}\right)$ is written as

$$
\begin{aligned}
\varphi_{\mathcal{N}\left(\mu, \sigma^{2}\right)}(t) & =\exp \left\{i \mu t-\frac{\sigma^{2} t^{2}}{2}\right\} \\
& =\left[\exp \left\{i \frac{\mu}{n} t-\frac{\sigma^{2} / n}{2} t^{2}\right\}\right]^{n} \\
& =\left[\varphi_{\mathcal{N}\left(\mu / n, \sigma^{2} / n\right)}(t)\right]^{n},
\end{aligned}
$$

as $n$th power of characteristic function of a normal distribution $\mathscr{N}\left(\mu / n, \sigma^{2} / n\right)$.

Example 4. The characteristic function of Cauchy distribution $C(c)$ is written as

$$
\varphi_{C(c)}(t)=\exp \{-c|t|\}=\left[\exp \left(-\frac{c}{n}|t|\right)\right]^{n}=\left[\varphi_{\mathscr{C}(c / n)}(t)\right]^{n},
$$

as $n$th power of the characteristic function of a Cauchy distribution $\mathscr{C}(c / n)$.

Example 5. The characteristic function of Poisson distribution $P(\lambda)$ is written as

$$
\begin{aligned}
\varphi_{\mathscr{P}(\lambda)}(t) & =\exp \left\{\lambda\left(e^{i t}-1\right)\right\} \\
& =\left[\exp \left\{\frac{\lambda}{n}\left(e^{i t}-1\right)\right\}\right]^{n} \\
& =\left[\varphi_{\mathscr{P}(\lambda / n)}(t)\right]^{n},
\end{aligned}
$$

as $n$th power eme of the characteristic function of a Poisson law $\mathscr{P}(\lambda / n)$.

Example 6. The characteristic function of Gamma distribution $\Gamma(r, \lambda)$ is written as

$$
\varphi_{-(r, \lambda)}(t)=\frac{1}{(1-i t / \lambda)^{r}}=\left[\frac{1}{(1-i t / \lambda)^{r / n}}\right]^{n}=\varphi_{-(r / n, \lambda)}(t),
$$

as $n$th power eme of characteristic function of a Gamma distribution $-(r / n, \lambda)$.

The same is true for the exponential law $(\operatorname{Exp}(\lambda)=\Gamma(1$, $\lambda)$ ) and the law of $\left.\chi^{2}=\Gamma(n / 2,1 / 2)\right)$.
Remark 7. A mixture of finished normal distributions is not divisible infinitely.

Theorem 8. A random variable $X$ is the limit of a sum of $n$ random variables i.i.d. if and only if $X$ is divisible indefinitely.

Proof. The demonstration was detailed in Shiryayev [9].

Definition 9. A random variable $X$ is stable if for $X_{1}, X_{2}$ two independent random variables that have same law as $X$ and any two real positive constants $a, b$, then

$$
a X_{1}+b X_{2}=c X+d \text {, in distribution }
$$

satisfies for some $c \in \mathbb{R}^{+}$and $d \in \mathbb{R}$.

Definition 10. A random variable $X$ is stable distribution if and only if, for all $n$ and any independent identique family $X_{1}, X_{2}, \ldots, X_{n}$ which have the same law that $X$, there is $a_{n}>0$ and $b_{n} \in R$, as

$$
X_{1}+X_{2}+\cdots+X_{n} \stackrel{d}{=} a_{n} X+b_{n} .
$$

Proposition 11. If $X$ is stable, $X$ is divisible indefinitely. The inverse is false (see the example below, the Poisson distribution).

Proof. Just take the random variables $Y_{i}=\left(X_{i}-b_{n} / n\right) / a_{n}, i=$ $1, \ldots, n$.

As $X_{i}$ are independent, $Y_{i}$ are also independent, and by substitution, we have

$$
Y_{1}+Y_{2}+\cdots+Y_{n} \stackrel{d}{=} X
$$

Remark 12. This definition is coinside of the central limit theorem if $X$ is following a normal law.

Theorem 13. $X$ has domain of attraction; that is, there is a sequence of random variables i.i.d. $\left(X_{i}\right)_{i \in N}$, a sequence of positive real numbers $\left(a_{i}\right)_{i \in \mathbb{N}}$, and a sequence of real numbers $\left(b_{i}\right)_{i \in \mathbb{N}}$, such as

$$
\frac{1}{a_{n}} \sum_{i=1}^{n} X_{i}-b_{n} \longrightarrow X, \text { in distribution. }
$$

Proof. The demonstration is detailed in Shiryayev [9].

Corollary 14 (Levy-Khinchin). The characteristic function of a stable random variable $X$ admits the following form:

$$
\begin{aligned}
& \psi_{X}(t) \\
& =\left\{\begin{array}{l}
\exp \left[-\sigma^{\alpha}|t|^{\alpha}\left(1-i \beta \cdot \operatorname{sgn}(t) \cdot \operatorname{tg}\left(\frac{\alpha \pi}{2}\right)\right)+i \mu t\right], \\
\exp \left[-\sigma|t|\left(1+\frac{2}{\pi} i \beta \cdot \operatorname{sgn}(t) \cdot \ln |t|\right)+i \mu t\right], \\
\alpha \neq 1 \\
\alpha=1,
\end{array}\right.
\end{aligned}
$$

$$
\text { where } \operatorname{sgn}(t)= \begin{cases}1, & t>0 \\ 0, & t=0 \\ -1, & t<0\end{cases}
$$


Proof. The demonstration is detailed in Gnedenko and Kolmogorov [10].

The random variable $X$ is a stable law with parameters $\alpha$, $\beta, \sigma$, and $\mu$; we note that

$$
\begin{aligned}
& X \equiv S_{\alpha}(\beta, \sigma, \mu), \\
& \quad \text { where } 0<\alpha \leq 2,-1 \leq \beta \leq 1, \sigma \geq 0, \mu \in \mathbb{R} .
\end{aligned}
$$

But this representation of the FC, called parameterization standard, has the disadvantage of not being continued in all of its parameters. In fact, there is discontinuity in the points where $\alpha=1$ and $\beta=0$; otherwise, there are other parameterization of the FC more adapted to different problems. Consider

$$
\begin{aligned}
& \psi_{X}(t) \\
& =\left\{\begin{array}{lr}
\exp \left[-\sigma^{\alpha}|t|^{\alpha}\left(1+i \beta \cdot \operatorname{sgn}(t) \cdot \tan \frac{\alpha \pi}{2}\right)\left(|\sigma t|^{1-\alpha}-1\right)+i \mu_{0} t\right], \\
\alpha \neq 1 \\
\exp \left[-\sigma|t|\left(1+\frac{2}{\pi} i \beta \cdot \operatorname{sgn}(t) \cdot \ln |t|+\ln \sigma\right)+i \mu_{0} t\right], & \\
& \alpha=1 .
\end{array}\right.
\end{aligned}
$$

This representation of Zolotarev's $M$ parameterization notes $X \sim S_{\alpha}^{0}\left(\beta, \sigma, \mu_{0}\right)$. The parameters $\alpha, \beta$, and $\sigma$ are the same as those of the standard parameterization, but $\mu$ and $\mu_{0}$ are related by

$$
\mu= \begin{cases}\mu_{0}-\beta \sigma \tan \frac{\alpha \pi}{2}, & \alpha \neq 1 \\ \mu_{0}-\frac{2}{\pi} \beta \sigma \ln \sigma, & \alpha=1 .\end{cases}
$$

This parameterization is very important because the characteristic function and the cumulative distribution function are continuing in relation to the four parameters. $S_{0}$ is well conditioned numerically by calculation.

Another parameterization $S^{1}$ is given by the following:

$$
\begin{aligned}
& \psi_{X}(t) \\
& =\left\{\begin{array}{l}
\exp \left[-\sigma_{2}^{\alpha}|t|^{\alpha} \exp \left(-i \beta_{2} \cdot \operatorname{sgn}(t) \cdot \frac{\pi}{2} K(\alpha)\right)+i \mu t\right], \\
\exp \left[-\sigma_{2}|t|\left(\frac{\pi}{2}+i \beta_{2} \cdot \operatorname{sgn}(t) \cdot \ln |t|\right)+i \mu t\right], \begin{array}{r}
\alpha \neq 1 \\
\alpha=1,
\end{array}
\end{array}\right.
\end{aligned}
$$

where

$$
K(\alpha)=\alpha-1+\operatorname{sgn}(1-\alpha)= \begin{cases}\alpha, & \alpha \leq 1 \\ \alpha-2, & \alpha \geq 1\end{cases}
$$

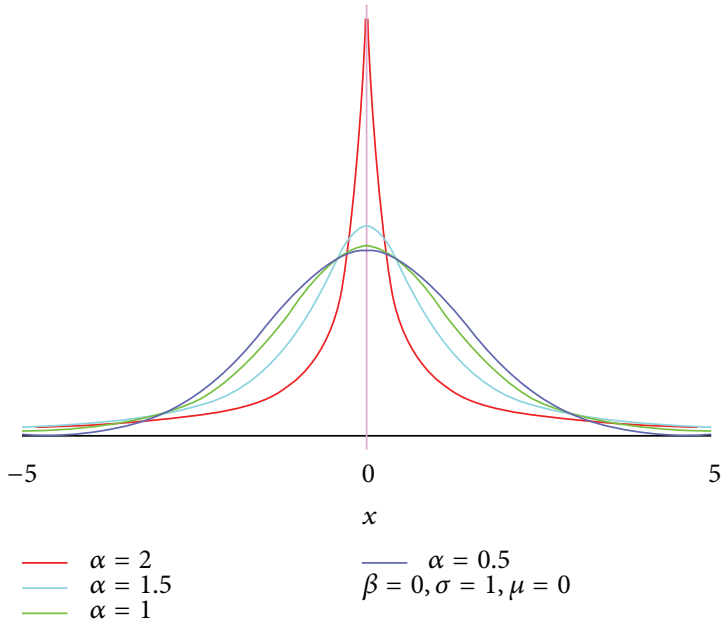

FIGURE 1: pdf plot for different values of alpha.

where parameters $\alpha$ and $\mu$ are the same as for standard parameterization; other parameters meet the following relationships:

$$
\begin{aligned}
\sigma_{2} & =\sigma\left(1+\beta^{2} \tan ^{2} \frac{\alpha \pi}{2}\right)^{1 / 2 \alpha}, \\
\tan \left(\beta_{2} \frac{\pi K(\alpha)}{2}\right) & =\beta \tan \frac{\alpha \pi}{2}, \quad \alpha \neq 1, \\
\sigma_{2} & =\frac{2 \sigma}{\pi}, \quad \beta_{2}=\beta, \quad \alpha=1 .
\end{aligned}
$$

Remark 15. The main disadvantage is that the densities of stable laws are unknown except in three cases.

(1) The Gaussian distribution $S_{2}(0, \sigma, \mu)$ where $f(x, 2,0$, $\sigma, \mu)=(1 / 2 \sigma \sqrt{\pi}) \exp \left(-(x-\mu)^{2} / 4 \sigma^{2}\right), x \in \mathbb{R}$.

(2) The Cauchy distribution $S_{1}(0, \sigma, \mu)$ where $f(x, 1,0$, $\sigma, \mu)=\left(2 \sigma / \pi\left((x-\mu)^{2}+4 \sigma^{2}\right)\right), x \in \mathbb{R}$.

(3) The Lévy distribution $S_{1 / 2}(1, \sigma, \mu)$ where $f(x, 1 / 2, \sigma$, $\mu)=(\sigma / 2 \pi)^{1 / 2}(x-\mu)^{3 / 2} \exp (-\sigma / 2(x-\mu)) \cdot I_{] \mu, \infty[}(x)$.

But since the implementation of the fast Fourier transform, stable densities are easy to calculate. We can approach this method density $f(x)=(1 / 2 \pi) \int_{-\infty}^{+\infty} e^{-i t x} \psi_{X}(t) d t$.

\subsection{Interpretation of the Parameters of the Characteristic Function of a Stable Law}

(1) The parameter $\alpha$ known as an exponent characteristic or a stability index describes the form of distribution or the degree of thickness of the tail distribution $(0<$ $\alpha \leq 2$ ). If $\alpha$ is smaller, then the tails of the distribution are thick. In other words, the more small $\alpha$ is, the more we see the existence of very large fluctuations (see Figure 1). A Gaussian distribution that has the maximum value of $\alpha$ is $\alpha=2$. 


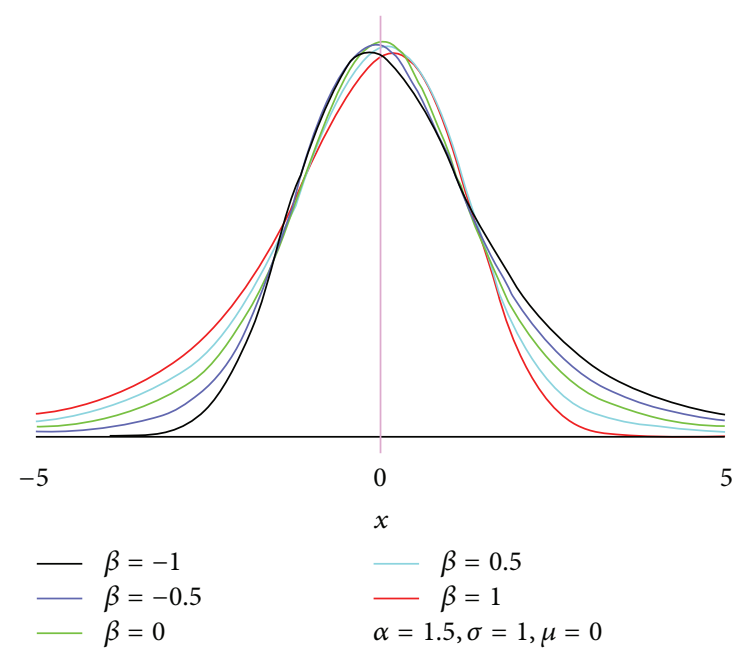

Figure 2: pdf plot for different values of beta.

(2) The parameter $\beta$ gives an idea of asymmetric distribution. This is the parameter of asymmetry. If $\beta$ is equal to -1 (resp., 1 ), distribution is totally asymmetrical left or right. When $\beta=0$, then the distribution is symmetric around $\mu$ (see Figure 2).

(3) The parameter $\sigma$ is called a scale factor. $\sigma$ is more grand, if more data are volatile (see Figure 3 ). The parameter $\sigma$ has the possibility to bend more or less the body of the distribution.

(4) The parameter $\mu$ location corresponds for $\alpha>1$ to the mean of the distribution. If $\beta=0$, then $\mu$ is the median. In other cases, $\mu$ cannot be interpreted.

2.2. Arithmetic Properties. Stable random variables $S_{\alpha}(\beta, \sigma$, $\mu$ ) have the following properties.

(i) $X_{1}, X_{2}$ are two independent random variables that follow, respectively, stable laws $S_{\alpha}\left(\beta_{1}, \sigma_{1}, \mu_{1}\right), S_{\alpha}\left(\beta_{2}\right.$, $\left.\sigma_{2}, \mu_{2}\right)$; then, $X_{1}+X_{2}$ follows a stable law $S_{\alpha}(\beta, \sigma, \mu)$ with

$$
\begin{aligned}
& \sigma=\left(\sigma_{1}^{\alpha}+\sigma_{2}^{\alpha}\right)^{1 / \alpha}, \\
& \beta=\frac{\beta_{1} \sigma_{1}^{\alpha}+\beta_{2} \sigma_{2}^{\alpha}}{\sigma_{1}^{\alpha}+\sigma_{2}^{\alpha}}, \\
& \mu=\mu_{1}+\mu_{2} .
\end{aligned}
$$

Note that, if $\beta_{1}=\beta_{2}$, then $\beta=\beta_{1}=\beta_{2}$.

(ii) If $X \sim S_{\alpha}(\beta, \sigma, \mu), a \in \mathbb{R}$, then $X+a \sim S_{\alpha}(\beta, \sigma, \mu+a)$.

(iii) If $X_{1}, X_{2}$ follows a stable law $S_{\alpha}(\beta, \sigma, \mu), a \in \mathbb{R}^{+}, b \in$ $\mathbb{R}^{+}, c \in \mathbb{R}$, then

$a X_{1}+b X_{2}+c \stackrel{d}{=} S_{\alpha}\left(\beta, \sigma\left(a^{\alpha}+b^{\alpha}\right)^{1 / \alpha}, \mu\left(a^{\alpha}+b^{\alpha}\right)^{1 / \alpha}+c\right)$,

where " $\stackrel{d}{=}$ " means "has the same distribution."

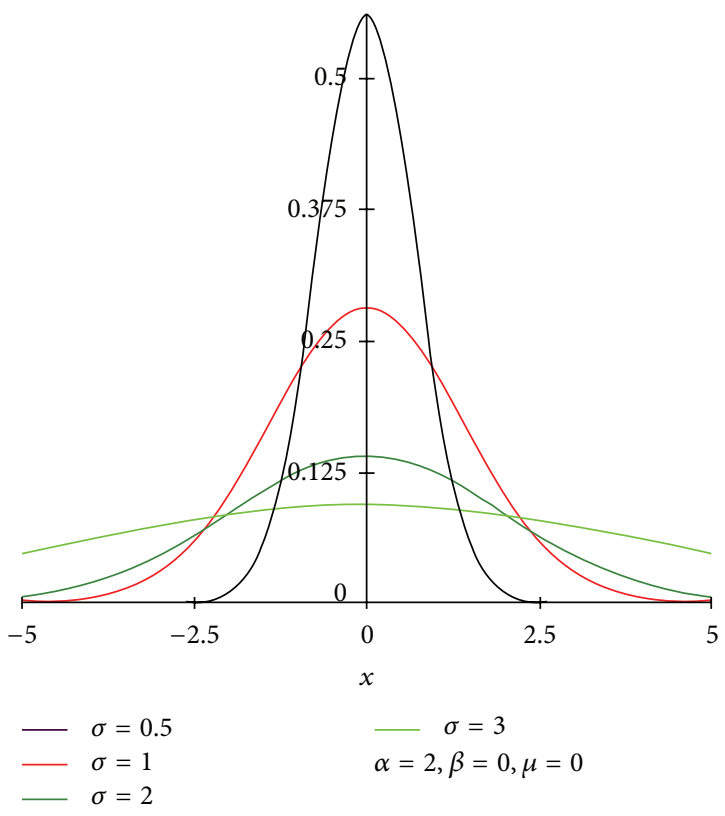

FIGURE 3: pdf plot for different values of sigma.

(iv) $X$ is a symmetric random variable around $\mu$ if and only if the law of $X$ is $S_{\alpha}(0, \sigma, \mu)$. We note that $S \alpha S$ is a symmetric stable random variable with $\mu=0$.

(v) Let $X$ be a random variable law $S_{\alpha}(\beta, \sigma, \mu)$ with $\alpha \epsilon$ $(0,2]$, then

$$
\begin{array}{ll}
E\left(|X|^{p}\right)<\infty & \text { if } p \in] 0 ; \alpha[, \\
E\left(|X|^{p}\right)=\infty & \text { if } p \in] \alpha ; \infty[.
\end{array}
$$

(vi) Let $X$ be a random variable law $S_{\alpha}(\beta, \sigma, \mu)$ with $\alpha \epsilon$ ] $1,2[$, then

$$
E(X)=\mu .
$$

In this section, it is assumed that the samples $X_{1}, X_{2}$, $\ldots, X_{n}$ are independent and equally distributed stable ran-

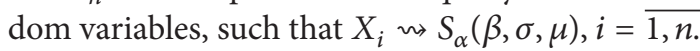

\subsection{The Asymptotic Behavior}

Theorem 16 (see [11]). For $0<\alpha<2,-1 \leq \beta \leq 1, \sigma \geq 0$, $\mu \in \mathbb{R}$, If $X \sim S_{\alpha}(\beta, \sigma, \mu)$, then

$$
\begin{aligned}
& \qquad \begin{aligned}
& \lim _{x \rightarrow \infty} x^{\alpha} P(X>x)=C_{\alpha}(1+\beta) \sigma^{\alpha}, \\
& \text { where } C_{\alpha}=\left(2 \int_{0}^{+\infty} x^{-\alpha} \sin (x) d x\right)^{-1} \\
&=\frac{1}{\pi} \Gamma(\alpha) \sin \left(\frac{\alpha \pi}{2}\right),
\end{aligned}
\end{aligned}
$$

by derivation, for large data,

$$
f(x, \alpha, \beta, \sigma, \mu) \sim \alpha \sigma^{\alpha}(1+\beta) \sin \left(\frac{\alpha \pi}{2}\right) \frac{\Gamma(\alpha)}{\pi} x^{-(\alpha+1)},
$$


or $\Gamma$ denotes the Gamma function defined by $\Gamma(\alpha)=$ $\int_{0}^{+\infty} e^{-t} t^{\alpha-1} d t$

As $x \rightarrow \infty, P(X>x)=C_{\alpha}(1+\beta) \sigma^{\alpha} x^{-\alpha} \Rightarrow F(x)=$ $P(x \leq x)=1-P(X>x)=1-C_{\alpha}(1+\beta) \sigma^{\alpha} x^{-\alpha}$, then by derivation $f(x)=(F(x))^{\prime}=\alpha C_{\alpha}(1+\beta) \sigma^{\alpha} x^{-\alpha-1}=\alpha C_{\alpha}(1+$ $\beta) \sigma^{\alpha} x^{-(\alpha+1)}$.

\subsection{Parameter Estimation}

2.4.1. Tail Exponent Estimation. There are many methods to give estimators of the index exponent of stable distributions, but we describe here the regression method, which is based on the effect that, for large enough values of $X$, the tails of stable laws come in a power of alpha; see (22); then, we consider a linear regression as

$$
\ln P(X>x) \sim \ln C_{\alpha}(1+\beta) \sigma^{\alpha}-\alpha \ln x,
$$

where the slope of the line will be an estimator of $-\alpha$.

2.5. Simulation of an $\alpha$-Stable Distribution. To simulate the stable laws, there is an algorithm developed by Chambers et al. [5]. It can generate a law $S_{\alpha}(\beta, 1,0)$; then to obtain a $S_{\alpha}(\beta, \sigma, \mu)$ stable law, just make a change of variables.

Step 1. Generate two random variables as follows.

Let $U$ be uniform on ]0, 1[. Then, $W=\pi U-(\pi / 2)$ is uniform on $]-\pi / 2, \pi / 2[$, and $Z=-\ln (1-U)$ is exponentially distributed with mean 1.

Step 2. Generate $X$ a $S_{\alpha}(\beta, 1,0)$ law.

(i) For $\alpha \neq 1$

$$
\begin{aligned}
X= & \zeta+\frac{\sin \alpha W-\zeta \cos \alpha W}{(\cos W)^{1 / \alpha}} \\
& \times\left(\frac{\cos (1-\alpha) W-\zeta \sin (1-\alpha) W}{Z}\right)^{((1-\alpha) / \alpha)} .
\end{aligned}
$$

(ii) For $\alpha=1$

$$
X=\frac{2}{\pi}\left[\left(\frac{\pi}{2}+\beta W\right) \tan Y-\beta \ln \frac{(\pi / 2) \cos W}{(\pi / 2)+\beta W}\right],
$$

Step 3. Generate a $S_{\alpha}(\beta, \sigma, \mu)$ law using these transformations:

$$
\begin{gathered}
Y=\sigma X+\mu, \quad \text { if } \alpha \neq 1, \\
Y=\sigma X+\frac{2}{\pi} \beta \sigma \ln \sigma+\mu, \quad \text { if } \alpha=1 .
\end{gathered}
$$

For more details see Samorodnitsky and Taqqu, [12, page 43].

\section{Probability Ratio Test Applying to Stable Laws in the Case $x \rightarrow+\infty$}

We will use $\theta$ for the exponent characteristic and $\delta$ for the asymmetry parameter to avoid confusion with the symbols $\alpha$ and $\beta$ which were used in type I error and type II error in Wald's sequential probability ratio test, and for simplifying the process, let us consider symmetric standard alpha stable random variables $X_{i} \equiv S_{\theta}(0,1,0), i=1,2, \ldots, m$, in other terms $(\beta=0, \sigma=1, \mu=0)$ where $\theta$ is unknown.

We assume that we have a sample of $X_{1}, X_{2}, \ldots, X_{n}$ independent random variables which follow the same law $X \equiv$ $S_{\theta}(0,1,0)$ with unknown $\theta$.

We want to use the sequential probability ratio test SPRT, for the hypothesis

$$
\begin{aligned}
& H_{0}: \theta=\theta_{0}, \\
& \text { against the hypothesis, } \\
& H_{1}: \theta=\theta_{1},
\end{aligned}
$$

where $\theta_{1}>\theta_{0}>0$, if we want to get a test as $P$ (Rejects $H_{0} / H_{0}$ is true $)=\alpha$ and $P\left(\right.$ Accepts $H_{0} / H_{0}$ is false $)=\beta$.

We are willing to choose values for $\alpha$ and $\beta$ as the Wald's [7] approximation $A=(1-\beta) / \alpha, B=\beta /(1-\alpha)$. Where $\alpha, \beta \epsilon$ $[0.01 ; 0.05]$, at the end of the $m$ th observation:

(i) accept $H_{0}$ if $\sum_{i=1}^{m} Z_{i} \leq \ln B$;

(ii) accept $H_{1}$ if $\sum_{i=1}^{m} Z_{i} \geq \ln A$;

(iii) continue the test if $\ln A<\sum_{i=1}^{m} Z_{i}<\ln B$, with $Z_{i}$ defined by the relationship in general by

$$
\begin{aligned}
Z_{i} & =\ln \frac{f\left(x_{i}, \theta_{1}, \delta, \sigma, \mu\right)}{f\left(x_{i}, \theta_{0}, \delta, \sigma, \mu\right)} \\
& =\ln \frac{\theta_{1} \sigma^{\theta_{1}}(1+\delta) \sin (\alpha \pi / 2)(\Gamma(\alpha) / \pi) x^{-\left(\theta_{1}+1\right)}}{\theta_{0} \sigma^{\theta_{0}}(1+\delta) \sin (\alpha \pi / 2)(\Gamma(\alpha) / \pi) x^{-\left(\theta_{0}+1\right)}} .
\end{aligned}
$$

In our case of symmetric standard alpha stable random variables, we have

$$
\begin{aligned}
Z_{i} & =\ln \frac{f\left(x_{i}, \theta_{1}, 0,1,0\right)}{f\left(x_{i}, \theta_{0}, 0,1,0\right)} \\
& \sim \ln \frac{\theta_{1} \sin \left(\theta_{1} \pi / 2\right)\left(\Gamma\left(\theta_{1}\right) / \pi\right) x_{i}^{-\left(\theta_{1}+1\right)}}{\theta_{0} \sin \left(\theta_{0} \pi / 2\right)\left(\Gamma\left(\theta_{0}\right) / \pi\right) x_{i}^{-\left(\theta_{0}+1\right)}},
\end{aligned}
$$

so,

$$
\begin{aligned}
& Z_{i}=\ln \left(\frac{\theta_{1} \Gamma\left(\theta_{1}\right) \sin \left((1 / 2) \pi \theta_{1}\right)}{\theta_{0} \Gamma\left(\theta_{0}\right) \sin \left((1 / 2) \pi \theta_{0}\right)} x_{i}^{\theta_{0}-\theta_{1}}\right) \\
& Z_{i}=\ln \frac{\theta_{1}}{\theta_{0}}+\ln \frac{\Gamma\left(\theta_{1}\right)}{\Gamma\left(\theta_{0}\right)}+\ln \frac{\sin \left((1 / 2) \pi \theta_{1}\right)}{\sin \left((1 / 2) \pi \theta_{0}\right)}+\left(\theta_{0}-\theta_{1}\right) \ln x_{i}
\end{aligned}
$$

In (30), we note that for $1<\theta<2, \sin ((1 / 2) \pi \theta)>0$, then

$$
\begin{aligned}
\sum_{i=1}^{m} Z_{i} \sim m & \ln \frac{\theta_{1}}{\theta_{0}}+m \ln \frac{\sin \left(\theta_{1} \pi / 2\right)}{\sin \left(\theta_{0} \pi / 2\right)} \\
& +m \ln \frac{\Gamma\left(\theta_{1}\right)}{\Gamma\left(\theta_{0}\right)}+\left(\theta_{0}-\theta_{1}\right) \sum_{i=1}^{m} \ln \left(x_{i}\right) .
\end{aligned}
$$


To summarize, we accept $H_{0}$ if

$$
\begin{gathered}
m \ln \frac{\theta_{1}}{\theta_{0}}+m \ln \frac{\sin \left(\theta_{1} \pi / 2\right)}{\sin \left(\theta_{0} \pi / 2\right)}+m \ln \frac{\Gamma\left(\theta_{1}\right)}{\Gamma\left(\theta_{0}\right)} \\
+\left(\theta_{0}-\theta_{1}\right) \sum_{i=1}^{m} \ln \left(x_{i}\right) \leq \ln B=\ln \frac{\beta}{1-\alpha} \\
\Longleftrightarrow \sum_{i=1}^{m} \ln \left(x_{i}\right) \leq \frac{1}{\left(\theta_{1}-\theta_{0}\right)} \\
\times\left(m \ln \frac{\theta_{1}}{\theta_{0}}+m \ln \frac{\sin \left(\theta_{1} \pi / 2\right)}{\sin \left(\theta_{0} \pi / 2\right)}\right. \\
\left.+m \ln \frac{\Gamma\left(\theta_{1}\right)}{\Gamma\left(\theta_{0}\right)}-\ln \frac{\beta}{1-\alpha}\right) \stackrel{\text { def }}{=} a_{m} .
\end{gathered}
$$

(i) Accept $H_{1}$, if

$$
\begin{aligned}
\sum_{i=1}^{m} Z_{i} \sim m \ln \frac{\theta_{1}}{\theta_{0}} & +m \ln \frac{\sin \left(\theta_{1} \pi / 2\right)}{\sin \left(\theta_{0} \pi / 2\right)}+m \ln \frac{\Gamma\left(\theta_{1}\right)}{\Gamma\left(\theta_{0}\right)} \\
& +\left(\theta_{0}-\theta_{1}\right) \sum_{i=1}^{m} \ln \left(x_{i}\right) \geq \ln A=\ln \frac{1-\beta}{\alpha} \\
\Longleftrightarrow \sum_{i=1}^{m} \ln \left(x_{i}\right) \geq & \frac{1}{\left(\theta_{1}-\theta_{0}\right)} \\
& \times\left(m \ln \frac{\theta_{1}}{\theta_{0}}+m \ln \frac{\sin \left(\theta_{1} \pi / 2\right)}{\sin \left(\theta_{0} \pi / 2\right)}\right. \\
& \left.\quad+m \ln \frac{\Gamma\left(\theta_{1}\right)}{\Gamma\left(\theta_{0}\right)}-\ln \frac{1-\beta}{\alpha}\right) \stackrel{\text { def }}{=} r_{m} .
\end{aligned}
$$

(ii) We take an additional observation if

$$
a_{m}<\sum_{i=1}^{m} \ln \left(x_{i}\right)<r_{m}
$$

In practice, we trace the following two parallel lines:

$$
\begin{aligned}
L_{0}: y= & \frac{1}{\left(\theta_{1}-\theta_{0}\right)} \\
& \times\left(m \ln \frac{\theta_{1}}{\theta_{0}}+m \ln \frac{\sin \left(\theta_{1} \pi / 2\right)}{\sin \left(\theta_{0} \pi / 2\right)}\right. \\
& \left.+m \ln \frac{\Gamma\left(\theta_{1}\right)}{\Gamma\left(\theta_{0}\right)}-\ln \frac{\beta}{1-\alpha}\right),
\end{aligned}
$$

$$
\begin{aligned}
L_{1}: y= & \frac{1}{\left(\theta_{1}-\theta_{0}\right)} \\
& \times\left(m \ln \frac{\theta_{1}}{\theta_{0}}+m \ln \frac{\sin \left(\theta_{1} \pi / 2\right)}{\sin \left(\theta_{0} \pi / 2\right)}\right. \\
& \left.+m \ln \frac{\Gamma\left(\theta_{1}\right)}{\Gamma\left(\theta_{0}\right)}-\ln \frac{1-\beta}{\alpha}\right) .
\end{aligned}
$$

Then we place on the same graph the points of coordinates $\left(m, \sum_{i=1}^{m} \ln \left(x_{i}\right)\right)$ as long as they are in the band plan defined by two straight, we decides $H_{0}$ whether $L_{0}$ is crossed the band formed by the two straights, and we decides $H_{1}$ if $L_{1}$ is crossed. We continue the sampling if the points are in the band.

Example 17. For a sample of $X_{1}, X_{2}, \ldots, X_{n}$ independent random variables which follow the same law $X \equiv S_{\theta}(0,1,0)$ with unknown $\theta$, we want to use the sequential probability ratio test $S P R T$, for the hypothesis

$$
H_{0}: \theta=\theta_{0}=1.3
$$

against the hypothesis,

$H_{1}: \theta=\theta_{1}=1.5$,

where $\theta_{1}>\theta_{0}>0$. Let $\alpha=0.01$ and $\beta=0.05$, then $A=(1-\beta) / \alpha=(1-0.05) / 0.01=95.0, B=\beta /(1-\alpha)=$ $0.05 /(1-0.01)=5.0505 \times 10^{-2}$.

$\ln A=\ln (95.0)=4.5539$ and $\ln B=\ln (5.0505 \times$ $\left.10^{-2}\right)=-2.9857$

$L_{0}: y=-0.50360 m+14.929$ with thick black line (straight).

$L_{1}: y=-0.50360 m-22.770$ with red line (straight) (see Figure 4).

Example 18. We assume that we have a sample of $X_{1}$, $X_{2}, \ldots, X_{n}$ independent random variables which follow the same normal distribution $N\left(\theta, \sigma^{2}\right)$ with unknown $\theta$. We want to use the sequential probability ratio test SPRT, for the hypothesis

$$
H_{0}: \theta=\theta_{0}
$$

against the hypothesis,

$$
H_{1}: \theta=\theta_{1} \text {, }
$$

where $\theta_{1}>\theta_{0}>0$; if we follow the same steps, we obtain

$$
\begin{aligned}
& L_{0}: y=\frac{\theta_{1}+\theta_{0}}{2} m+\frac{\sigma^{2}}{\theta_{1}-\theta_{0}} \ln \left(\frac{\beta}{1-\alpha}\right), \\
& L_{1}: y=\frac{\theta_{1}+\theta_{0}}{2} m+\frac{\sigma^{2}}{\theta_{1}-\theta_{0}} \ln \left(\frac{1-\beta}{\alpha}\right) .
\end{aligned}
$$

\subsection{Efficiency Function and Average Sample Number Function}

Definition 19. The efficiency function of the probability ratio test is

$$
\theta \longrightarrow \mathbb{P}_{\theta}\left(\text { Accepts } H_{0}\right) \text {. }
$$




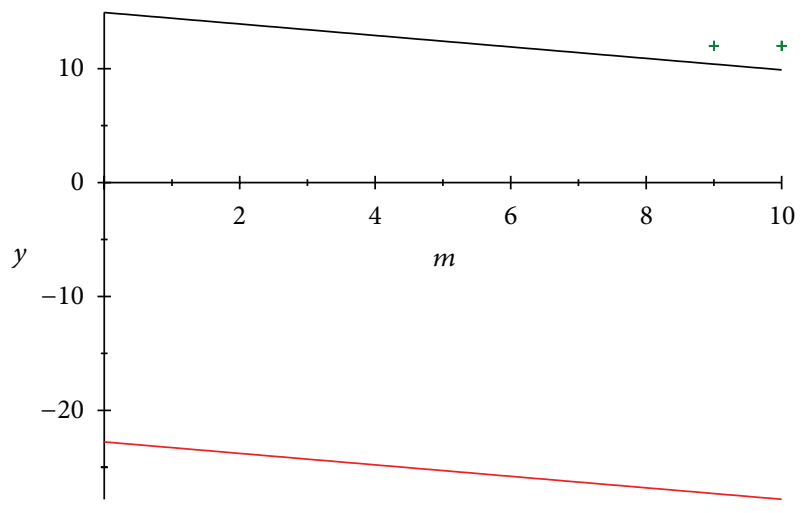

FIgUre 4: Accepting $H_{0}$.

It is possible to obtain additional information on the sequential probability test, especially on the number of observations $N$ needed to take decision, which is a discrete random variable that admits moments of all kinds; consider the function average sample number (ASN).

Definition 20. One calls the following average sample number (ASN) function:

$$
\theta \longrightarrow \mathbb{E}_{\theta}(N)
$$

Theorem 21. Let a fixed $\theta \in \Theta$. If $\mathbb{E}_{\theta}\left(\left|Z_{1}\right|\right)<+\infty$, then

$$
\mathbb{E}_{\theta}(N) \mathbb{E}_{\theta}\left(Z_{1}\right)=\mathbb{E}_{\theta}\left(S_{N}\right),
$$

where $S_{N}=\sum_{i=1}^{N} Z_{i}$ and $Z_{i}$ is defined by (29).

Theorem 22. Let a fixed $\theta \in \Theta$. If $\mathbb{E}_{\theta}\left(\left|Z_{1}\right|\right)<+\infty$ and $\mathbb{E}_{\theta}\left(Z_{1}\right) \neq 0$, then

$$
\mathbb{E}_{\theta}(N) \sim \frac{(1-L(\theta)) \ln A+L(\theta) \ln B}{\mathbb{E}_{\theta}\left(Z_{1}\right)} .
$$

In the case of stable densities with $1<\alpha<2, \mathbb{E}(X)=\mu<$ $+\infty$, see (v) in arithmetic properties,

$$
\begin{aligned}
& \mathbb{E}_{\theta}\left(\left|Z_{1}\right|\right) \\
& =\mathbb{E}_{\theta}\left(\mid \ln \frac{\theta_{1}}{\theta_{0}}+\ln \frac{\Gamma\left(\theta_{1}\right)}{\Gamma\left(\theta_{0}\right)}+\ln \frac{\sin \left((1 / 2) \pi \theta_{1}\right)}{\sin \left((1 / 2) \pi \theta_{0}\right)}\right. \\
& \left.\quad+\left(\theta_{0}-\theta_{1}\right) \ln x_{1} \mid\right) \\
& =\ln \frac{\theta_{1}}{\theta_{0}}+\ln \frac{\Gamma\left(\theta_{1}\right)}{\Gamma\left(\theta_{0}\right)}+\ln \frac{\sin \left((1 / 2) \pi \theta_{1}\right)}{\sin \left((1 / 2) \pi \theta_{0}\right)}+\left(\theta_{0}-\theta_{1}\right) \mu<\infty,
\end{aligned}
$$

and with a simple calculation, and for symmetric standard alpha stable random variable $(\beta=0, \sigma=1, \mu=0)$,

$$
\mathbb{E}_{\theta}\left(Z_{1}\right)=\ln \frac{\theta_{1}}{\theta_{0}}+\ln \frac{\Gamma\left(\theta_{1}\right)}{\Gamma\left(\theta_{0}\right)}+\ln \frac{\sin \left((1 / 2) \pi \theta_{1}\right)}{\sin \left((1 / 2) \pi \theta_{0}\right)} .
$$

Then,

$$
\mathbb{E}_{\theta}(N) \sim \frac{(1-L(\theta)) \ln A+L(\theta) \ln B}{\mathbb{E}_{\theta}\left(Z_{1}\right)},
$$

where $L(\theta)$ is the function of efficiency. With substitution in (29)

$$
\mathbb{E}_{\theta}\left(Z_{1}\right)=\mathbb{E}_{\theta_{0}}\left(Z_{1}\right)=\ln \frac{\theta_{1}}{\theta_{0}}+\ln \frac{\Gamma\left(\theta_{1}\right)}{\Gamma\left(\theta_{0}\right)}+\ln \frac{\sin \left((1 / 2) \pi \theta_{1}\right)}{\sin \left((1 / 2) \pi \theta_{0}\right)},
$$

finally, under $H_{0}$

$$
\begin{aligned}
& \mathbb{E}_{\theta_{0}}(N) \\
& \sim \frac{\alpha \ln ((1-\beta) / \alpha)+(1-\alpha) \ln (\beta /(1-\alpha))}{\ln \left(\theta_{1} / \theta_{0}\right)+\ln \left(\Gamma\left(\theta_{1}\right) / \Gamma\left(\theta_{0}\right)\right)+\ln \left(\sin \left((1 / 2) \pi \theta_{1}\right) / \sin \left((1 / 2) \pi \theta_{0}\right)\right)},
\end{aligned}
$$

because $L\left(\theta_{0}\right)=1-\alpha$, and under $H_{1}$

$$
\begin{aligned}
& \mathbb{E}_{\theta_{1}}(N) \\
& \sim \frac{(1-\beta) \ln ((1-\beta) / \alpha)+\beta \ln (\beta /(1-\alpha))}{\ln \left(\theta_{1} / \theta_{0}\right)+\ln \left(\Gamma\left(\theta_{1}\right) / \Gamma\left(\theta_{0}\right)\right)+\ln \left(\sin \left((1 / 2) \pi \theta_{1}\right) / \sin \left((1 / 2) \pi \theta_{0}\right)\right)},
\end{aligned}
$$

because $L\left(\theta_{1}\right)=\beta$.

Example 23. Let $\alpha=0.01$ and $\beta=0.05$; then, for the index parameter of symmetric stable distributions $\theta_{1}=1.5, \theta_{0}=$ 1.3 ,

$$
\mathbb{E}_{\theta_{0}}(N)=28.907
$$

In the case of normal distribution $\alpha=2$, that is, $\mathcal{N}(\theta, 1)$, we use the same steps, but here we have the analytic form of the density function, and the average sample number function is under $\mathrm{H}_{0}$

$$
\mathbb{E}_{\theta}(N) \sim \frac{\alpha \ln ((1-\beta) / \alpha)+(1-\alpha) \ln (\beta /(1-\alpha))}{-(1 / 2)\left(\theta_{1}-\theta_{0}\right)^{2}},
$$

because $L\left(\theta_{0}\right)=1-\alpha$, and under $H_{1}$

$$
\mathbb{E}_{\theta}(N) \sim \frac{(1-\beta) \ln ((1-\beta) / \alpha)+\beta \ln (\beta /(1-\alpha))}{(1 / 2)\left(\theta_{1}-\theta_{0}\right)^{2}},
$$

because $L\left(\theta_{1}\right)=\beta$, where

$$
\mathbb{E}_{\theta}\left(Z_{1}\right)=\left(\theta_{1}-\theta_{0}\right) \theta-\frac{1}{2}\left(\theta_{1}^{2}-\theta_{0}^{2}\right) .
$$

\section{Conclusion}

We use the fact that the tails of the Lévy-stable distributions are asymptotically equivalent to a Pareto law when $1<\alpha<2$ for large data as $x \rightarrow \infty, P(X>x)=1-F(x) \rightarrow$ $\sigma^{\alpha} C_{\alpha}(1+\beta) x^{-\alpha}$, where $C_{\alpha}=\left(2 \int_{0}^{\infty} x^{-\alpha} \sin x d x\right)^{-1}=$ $(1 / \pi) \Gamma(\alpha) \sin (\alpha \pi / 2)$ to obtain the probability ratio, and we 
compare the fit of two Pareto stable models using the sequential probability ratio test which is not possible in the other cases because of the lack of the analytical formulas of stable densities; then, we apply the theorem giving the average sample number function.

Can one give a bound of the average sample number function of stable distributions in our case, and in other cases?

Can one say that this amount of data is sufficient to make the decision that this population follows this stable law with this alpha parameter or the other laws with the second alpha parameter?

One can find many applications of the Pareto stable distributions in many fields when the data are very large, such as the flow of profits or revenues in public and private companies with huge capital, such as banks, insurance companies, and oil companies. Actuaries give speculation of instant transactions reliability and test hypotheses and advice to guide managers; however, it would be prudent in their decisions in cases where the data are incomplete in the sense that the mean of the number of data required to take decision is not achieved. As in the likelihood, we use the log data, which simplifies the calculus.

\section{References}

[1] J. P. Nolan, "An algorithm for evaluating stable densities in Zolotarev's (M) parameterization," Mathematical and Computer Modelling, vol. 29, no. 10-12, pp. 229-233, 1999.

[2] J. P. Nolan, "Numerical calculation of stable densities and distribution functions," Communications in Statistics. Stochastic Models, vol. 13, no. 4, pp. 759-774, 1997.

[3] S. Rachev and S. Mittnik, Stable Paretian Models in Finance, John Wiley \& Sons, New York, NY, USA, 2000.

[4] S. Mittnik, T. Doganoglu, and D. Chenyao, "Computing the probability density function of the stable Paretian distribution," Mathematical and Computer Modelling, vol. 29, no. 10-12, pp. 235-240, 1999.

[5] J. M. Chambers, C. L. Mallows, and B. W. Stuck, "A method for simulating stable random variables," Journal of the American Statistical Association, vol. 71, no. 354, pp. 340-344, 1976.

[6] V. M. Zolotarev, One-Dimensional Stable Distributions, American Mathematical Society, Providence, RI, USA, 1986.

[7] A. Wald, Sequential Analysis, John Wiley \& Sons, New York, NY, USA, 1947.

[8] M. Raghavachari, "Operating characteristic and expected sample size of a sequentail probability ratio test for the simple exponential distribution," Calcutta Statistical Association Bulletin, vol. 14, pp. 65-73.

[9] A. N. Shiryayev, Probability, vol. 95 of Graduate Texts in Mathematics, Springer, New York, NY, USA, 1984.

[10] B. V. Gnedenko and A. N. Kolmogorov, Limit Distributions for Sums of Independent Random Variables, Translated from the Russian, annotated, and revised by K. L. Chung, J. L. Doob and P. L. Hsu, Addison-Wesley, Reading, Mass, USA, 1968.

[11] A. Janiki and A. Weron, Simulation and Chaotic Behavior of $\alpha$ Stable Stochastic Processes, Marcel Dekker, New York, NY, USA, 1994.

[12] G. Samorodnitsky and M. S. Taqqu, Stable Non-Gaussian Random Processes, Chapman \& Hall, New York, NY, USA, 1994. 


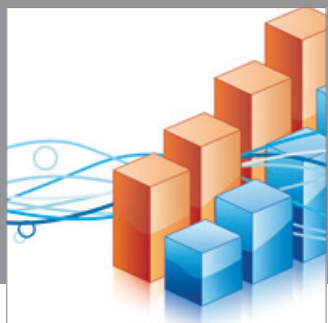

Advances in

Operations Research

mansans

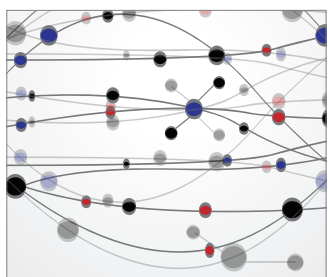

The Scientific World Journal
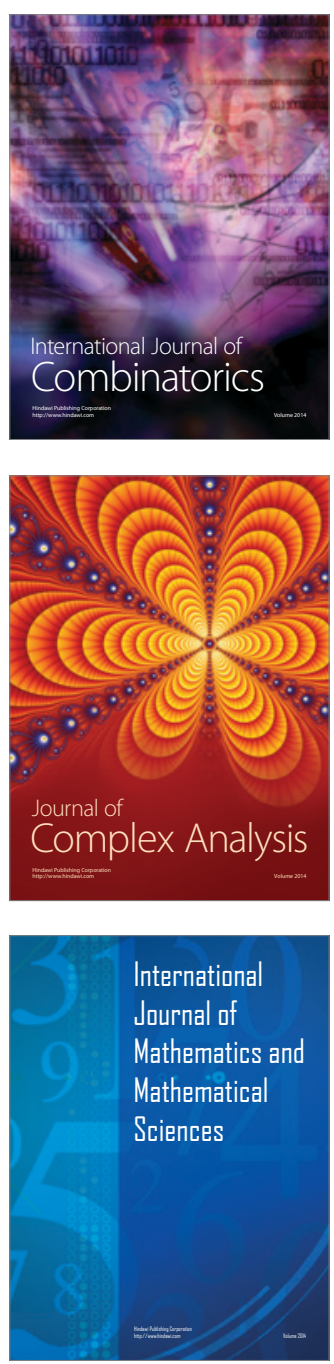
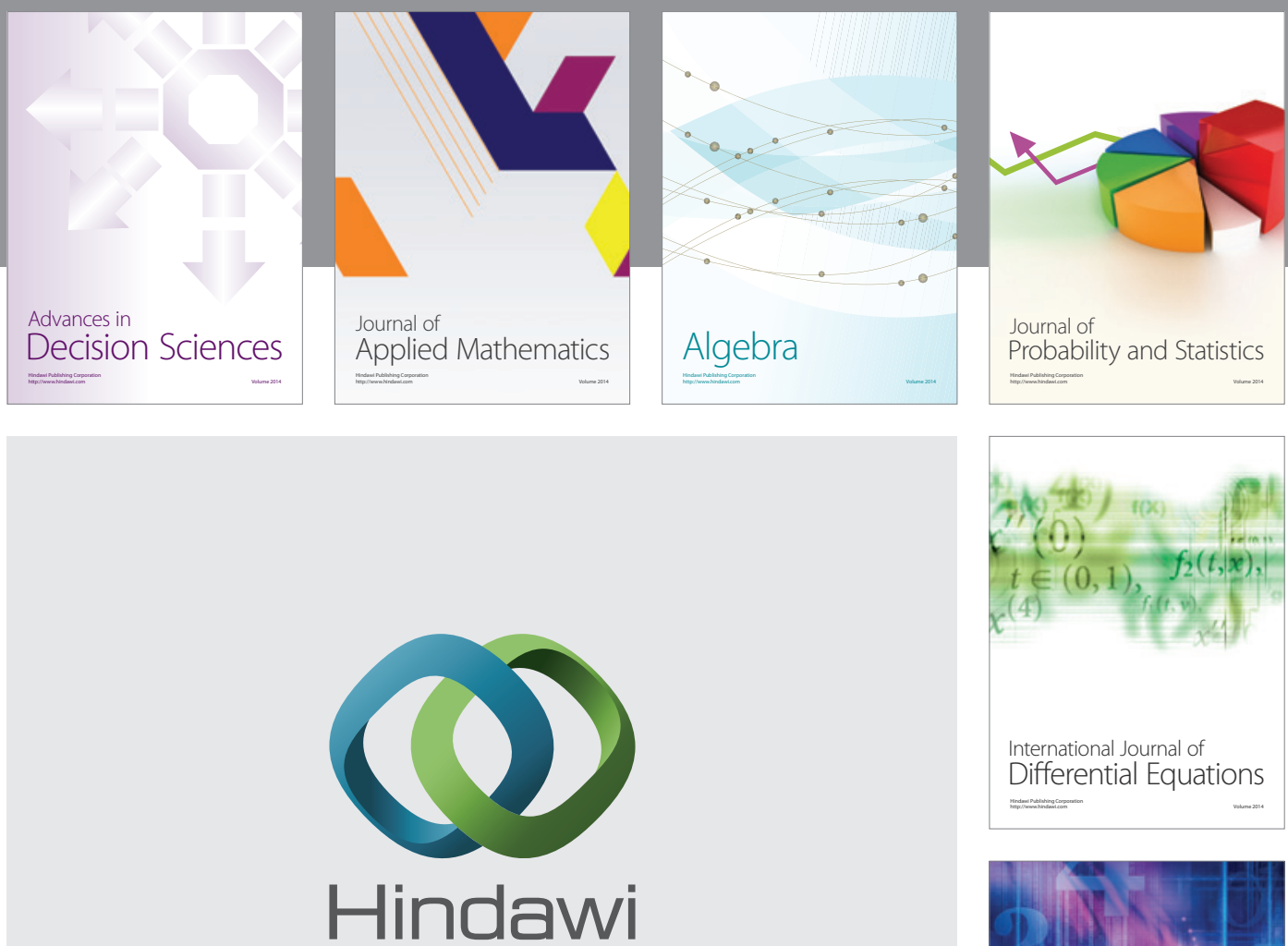

Submit your manuscripts at http://www.hindawi.com
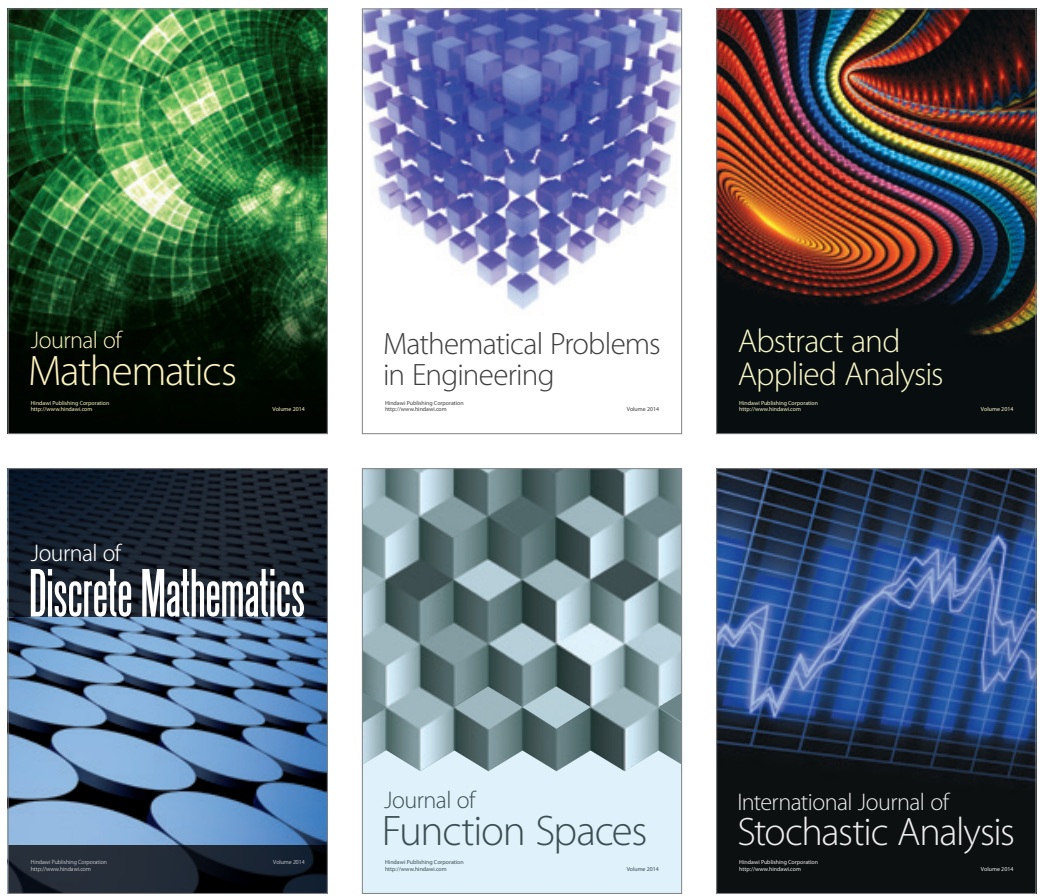

Journal of

Function Spaces

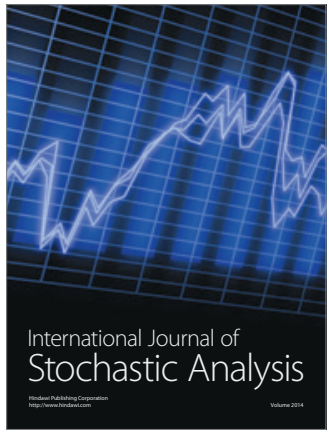

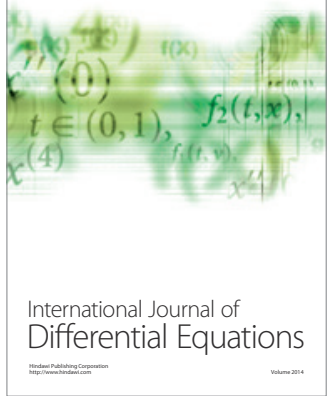
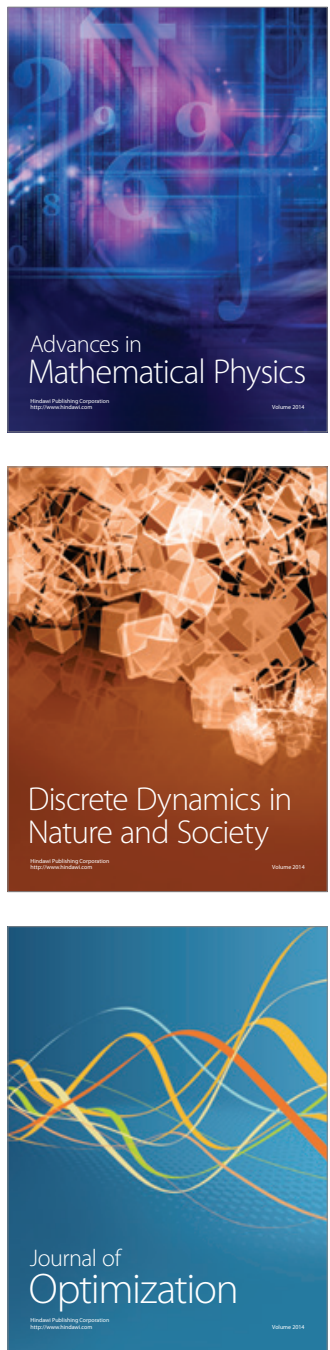